\title{
ENCRUCIJADAS PARA LAS SOCIEDADES PESQUERAS DEL SUR EN EL MARCO DE LA GLOBALIZACIÓN. LÍNEAS DE INVESTIGACIÓN Y PERSPECTIVAS TEÓRICO-METODOLÓGICAS
}

\author{
David Florido-Corral \\ Universidad de Sevilla \\ Grupo de Investigación GEISA
}

$\square$

\section{ACTUAL}

No ha sido resultado intencionado que la preparación final de este monográfico de la Revista Andaluza de Antropología dedicado a la Antropología Marítima y de la Pesca coincida con dos eventos políticos de calado que afectan a las pesquerías andaluzas: la reforma de la Política Pesquera Común y la reactivación de las rondas de negociación para un posible acuerdo pesquero entre Marruecos y la Unión Europea. En un marco histórico más amplio, el presente e inmediato futuro de las actividades pesqueras en las aguas meridionales se inscribe en una profunda transformación de la perspectiva, expectativas y estrategias de Europa respecto a la política marítima. Esta, la Política Marítima Integrada (2007), se ha convertido en la plataforma institucional más ambiciosa de la Unión Europa respecto del mar y las posibilidades de aprovechamiento económico del territorio y los recursos marítimos: energía (vientos, corrientes marinas y oleaje), acuicultura, turismo marítimo y costero, recursos minerales marinos (zinc, cobre, cobalto, boro, litio...) y biotecnología azul (aplicaciones farmacéuticas, cosméticas, alimentarias e industriales a partir de organismos marinos). Parece que la pesca se ha convertido en el patio trasero en el edificio institucional que desde Europa se viene construyendo respecto a las sociedades $\square$ 
litorales y los territorios marítimos. Y, sin embargo, los problemas tradicionales de este sector de actividad, en Andalucía y fuera de ella, persisten: la crisis de recursos en las pesquerías litorales; asociada a esta, la crisis institucional y los conflictos asociados entre modalidades pesqueras sobre territorios y recursos compartidos; o la competencia entre los sectores artesanal e industrial (incluyendo en este a determinadas formas de acuicultura), especialmente en territorios menos industrializados -pero que están en pleno proceso de industrialización de las pesquerías, ya sea con sus flotas o con flotas extranjeras-. Se trata de dinámicas que podremos ver ilustradas en el presente volumen. En este marco, se han puesto en marcha iniciativas desde distintos campos, y en las que la Antropología Marítima está participando, no sólo mediante acciones de investigación, sino mediante la incorporación comprometida en proyectos locales de sus investigadores. Un referente paradigmático en el ámbito patrimonial es la labor de Juan Luis Alegret, desde la Cátedra de Estudios Marítimos, respecto del Museo de la Pesca de Palamós (Girona, Cataluña), y que ahora ha venido a prolongarse en el Espai del Peix, experiencia que es ilustrada en este volumen. También ha sido muy destacada la labor de Antonio García Allut en Galicia, tanto para la puesta en marcha de una Reserva Marina de Os Miñarzos (Lira, Galicia), como para el desarrollo del proyecto cultural Mar de Lira o Lonxanet, que fue experiencia pionera en la comercialización directa de pesca artesanal. O también podemos destacar la labor de investigadores de la Universidad de La Laguna (Tenerife, Islas Canarias), con José Pascual a la cabeza, ilustrada en este monográfico mediante el trabajo relativo a la Reserva Marina Punta de La Restinga- Mar de las Calmas (La Restinga, El Hierro, Islas Canarias).

Un rasgo transversal de estas iniciativas ha sido la directa participación de los representantes de los pescadores, reproduciendo un modelo de gestión que cada vez ha encontrado más acomodo tanto en la teoría científica (Kooiman, 2008; Kooiman et al. 2005) como en los foros institucionales. Con todo, se trata de un proceso conflictivo, por cuanto no existen comunidades de pescadores como colectivos homogéneos en sus intereses, perspectivas y modos de actuación, y ni siquiera estos, los pescadores y sus familias, son ya ni el único ni el principal tejido social implicado en los asuntos marítimos y litorales.

En este marco, las contribuciones que el lector puede encontrar en este monográfico atraviesan tres procesos sociales que consideramos clave: i) las tensiones y conflictos por los recursos pesqueros entre lógicas productivas y culturales diferentes (podríamos simplificar este proceso a través del enfrentamiento artesanal $v s$. industrial); i) la participación de los representantes de los pescadores en la resolución de estos conflictos y en la puesta en marcha de soluciones para garantizar la sustentabilidad social de las pesquerías de menor escala; y iii) las dinámicas de patrimonialización de la cultura 
marítima, en distintas versiones, como una respuesta a este marco de crisis ${ }^{1}$. Desde luego, no son todas las posibles, y algunas de las líneas de investigación emergentes, como la del papel de las mujeres y la renovación social de las sociedades marítimo-pesqueras no está presente. Pero sí han sido recopiladas algunas de las que encontramos más interesantes para acercar al interesado en la materia a las aportaciones que el análisis antropológico y etno-histórico está haciendo en este campo de estudio.

\section{LA PARTICIPACIÓN DE LOS PESCADORES EN LA GESTIÓN COMO UNA GARANTÍA DE SOSTENIBILIDAD Y REPRODUCCIÓN SOCIAL DE LAS PESQUERÍAS}

Tanto los trabajos de José Pascual y Raquel Modino (Islas Canarias) como el de Susana Moreno y Juan Pablo Aris (Senegal) abordan la viabilidad de las reservas marinas como figuras de gestión de recursos y territorios pesqueros, incorporando las perspectivas locales, la fórmula de la cogestión y los principios de la gobernanza, entre los que podemos destacar la coordinación de la pluralidad de actores involucrados, la redefinición de las reglas que organicen la participación de los actores y la gestión de los recursos, la inclusión de criterios éticos y políticos en la gestión técnica, la retroalimentación de conocimientos y saberes (científico-técnicos y legos o vernáculos). Se trata de figuras que, tanto en el caso de España como en el resto del mundo, pueden estar referidas a objetivos muy diversos, como la gestión pesquera desde una perspectiva de sostenibilidad, la planificación turística, las actividades ambientales y de ocio, o las actividades científicas.

Más allá de la letra de las normas y los planes políticos, y de lo que los discursos institucionalizados expresan en la superficie, lo que la etnografía pone de manifiesto es la aparición de conflictos, tanto de las perspectivas, miradas e intenciones de los actores implicados, o que pueden resultar afectos por la declaración de esas figuras de gestión, como de las lógicas inmanentes de sus agencias (Estado, asociaciones de pescadores, colectivos ecologistas, científicos, etc.). En cualquier caso, lo que la antropología, con una amplísima bibliografía sobre el particular, sí puede dejar sentado es la necesidad de aplicar el enfoque del stakeholder approach (Jentoft, et al., 2012), los principios de la gobernanza interactiva (Kooiman et al. 2005) o de la ecología de saberes (Santos, 2007). Una aportación novedosa del trabajo de Pascual y Modino es la importancia del período inicial, la etapa inicial o zero step, en el que se prefigura la idea, se da a conocer y se buscan los mecanismos para ponerla en marcha, pues las herramientas usadas, la capacidad de involucrar a los potenciales afectados y la legitimidad de la agencia que impulsa el proceso serán decisivos para el éxito de la iniciativa. Lo que sí se puede

1. Este trabajo se inscribe dentro del proyecto de investigación del Plan Nacional de Investigación I+D+i Patrimonio Etnológico, Sociedad y Cultura Marítima, PESCUM (HAR 2010-15566), financiado por el Ministerio de Economía y Competitividad (2010-2013) 
concluir fehacientemente de la etnografía presentada es que la reserva marina canaria ha fortalecido a los pescadores frente al sector de ocio, servicios y turismo.

\section{PROCESOS DE PATRIMONIALIZACIÓN}

Los procesos de patrimonialización relativos a la cultura marítimo-pesquera pueden tener diversas vertientes, algunas de las cuales están reflejadas en este volumen. Una de estas posibilidades es la recuperación de la memoria histórica; es decir, el reconocimiento, vía investigación y difusión, del papel que los colectivos pesqueros han tenido en la vertebración social y cultural de un territorio, como hacen Rafael Cáceres y Ma Ángeles Corbacho respecto a las sociedades de pescadores en Andalucía. Otras veces, en un sentido más restringido, el reconocimiento puede servir para recuperar el papel que los movimientos obreros en el ámbito marítimo pesquero tuvieron en el obrerismo, en general, en los años veinte y treinta, a escala del Estado español, como pone de manifiesto el trabajo de Dionisio Pereira.

$\mathrm{Su}$ aportación, de sólida fundamentación empírica, representa un esfuerzo de descubrimiento del papel del asociacionismo obrero de inspiración anarquista en el mundo social marinero de Galicia, aportando sus nombres, sus estrategias, sus alianzas y enfrentamientos. Dar a conocer sus estrategias basadas en un modelo autónomo de acción directa y el uso sistemático de la huelga nos permite apreciar un nuevo paisaje sociológico a incorporar a la visión estereotipada de la marinería como colectivo, basada en el ensimismamiento, el solipsismo y teñida de un halo de atemporalidad preñado de romanticismo. Las iniciativas sindicales hicieron aflorar el conflicto latente que en el sector pesquero español se venía incubando desde finales del siglo XIX como consecuencia de su progresiva capitalización y del empeño de los armadores por traspasar a los trabajadores los costes de la modernización de sus buques.

Este ejercicio de reconocimiento pone de manifiesto cómo el asociacionismo anarquista pesquero-marítimo en Galicia sirvió como precursor de la estructuración cenetista en federaciones de industria a un nivel más amplio. Recuperar su recuerdo equivale a dar a conocer la diversidad de organizaciones, sus herramientas de difusión, que sirvieron de canalización de las reivindicaciones laborales y sociales de los trabajadores del mar, pero también de impulso a otros ramos productivos. Pero también el rescate de este episodio sirve para conocer el fracaso en los objetivos de movimiento social marítimo-pesquero, que se extendió por los litorales españoles. Los enfrentamientos entre organizaciones obreras (CNT-UGT) facilitaron el fracaso sindical a favor de la patronal de armadores, tanto en Galicia, como en el Sur. Las organizaciones sindicales, a pesar de sus escisiones internas, buscaban la constitución de una entidad unionista a nivel estatal, que hablase de tú a tú a la patronal de armadores, justo en el momento de la irrupción del alzamiento militar del verano de 1936, y para ello trasladaron la sede de la Federación Nacional de Industrias Pesqueras desde Galicia a Cádiz, pasando por Gijón, en 1934, poniendo de 
manifiesto un rasgo secular de la cultura y sociología marítimas: la intensidad de flujos entre distintos territorios.

Dar a conocer estos flujos y movimientos para el territorio andaluz ha sido precisamente el objetivo fundamental del trabajo de Cáceres y Corbacho sobre Andalucía -aunque también el trabajo de Saavedra en Chile ha constatado la continuidad histórica de las migraciones entre zonas costeras alejadas geográficamente: movimientos que garantizan el trasvase de pautas culturales, configurando una tradición híbrida de reconfiguraciones culturales, como en el caso andaluz-. Ya no se trata de redescubrir procesos institucionales, más brillantes, con mayor proyección política, sino de la silente transmisión de prácticas, técnicas, léxicos, saberes y conocimientos desde unos a otros territorios, como consecuencia de los movimientos poblacionales inter e intraterritoriales. Ello nos permite apreciar lo inadecuado de las imágenes al uso sobre la identidad cultural, socioeconómica y territorial de Andalucía, y cómo podemos recrear una imagen vertebrada a partir de los distintos entornos litorales ibéricos, atlántico, cantábrico y mediterráneo; o entre España y el Norte de África; o entre Italia y todo el ámbito Mediterráneo hispánico, hasta las costas portuguesas. La reinvención de la historia de Andalucía, recuperando del olvido las sociedades litorales y sus culturas productivas, para insertar en su texto las experiencias marítimas, es una tarea pendiente que reclama este trabajo, como habían reivindicado diversos autores hace una década (Monográfico del Boletín PH, 'Reconocer el Patrimonio Pesquero', 44, 2003).

La patrimonialización de la cultura pesquera puede tener consecuencias más pragmáticas, como se pone de manifiesto en el trabajo de Juan Luis Alegret sobre la experiencia del Espai del Peix en la cofradía de pescadores de Palamós (Girona). Se trata de una propuesta de valoración patrimonial con varios ejes (gastronomía, turismo en zonas portuarias, reconocimiento de las pesquerías tradicionales en el entorno local), que complementa el Museo de la Pesca. La propuesta se basa en la ampliación del patrimonio al pescado de poco precio. Así, su alcance excede a las experiencias más convencionales: porque se extiende a elementos culturales que no habían sido tratados ni pensados patrimonialmente por la sociedad local -bien representada por la exitosa pesquería, en lo económico, de la gamba roja-; y porque significa una apuesta que afecta al mismo tiempo a elementos naturales y culturales.

Se trata de una iniciativa de patrimonialización entendida como singularización y valorización -tanto económica como social-, y que le sirve al autor para poner de manifiesto las paradojas de estas iniciativas, pues la revaloración económica puede desembocar en un ralo mercantilismo, y la resignificación social, si no se hace con la participación de los actores locales y desde sus perspectivas, puede reproducir una suerte de tecnocracia cientifista. 
Haciéndose eco del aforismo de Josep Pla -la cocina es el paisaje puesto en la cazuelael Espai del Peix presenta el pescado en un contexto recreado que permite el acceso a conocimientos, técnicas e historias pesqueras locales: permite paladear la maritimidad, con un halo de exotización, porque las especies objeto de patrimonialización son aquellas que sólo son conocidas en los entornos locales, quedando segregadas de los circuitos regionales y más amplios. Y porque se trata de prácticas en proceso de desaparición como consecuencia de las transformaciones en las estrategias de pesca de los pescadores en la actualidad. Son especies que se habían caracterizado por su bajo valor económico, pero que gracias a su alto valor identitario, pueden alcanzar un precio considerable como consecuencia del turismo experiencial. Se trata por tanto de un excelente referente para indagar cómo los procesos sociales generan valor económico, al margen de las supuestas leyes de la oferta y la demanda y de la maximización racionalizadora del homo oeconomicus.

\section{4. ¿QUÉ ESPACIO PARA LAS PESQUERÍAS ARTESANALES EN EL NUEVO MARCO GLOBAL?}

Tanto los estudios presentados sobre Chile y Senegal, acerca de las amenazas que han de arrostrar las pesquerías artesanales, ponen de manifiesto el impacto que los sistemas locales reciben de flujos de poder y capital, líquidamente ubicuos, pero que aterrizan en sus territorios tradicionales. Estos trabajos nos obligan a plantearnos el papel del Estado en el concierto geo-económico mundial. Parece indudable que el Estado nacional ha ido perdiendo parte de su capacidad de influencia, y que, desde los años setenta del siglo XX a esta parte, se ha ido configurando un nuevo orden geoeconómico-político mundial (Agnew, 2003; Power \& Campbell, 2010). Wallerstein (2004) describe el sistema mundial actual como una realidad social configurada mediante acuerdos internacionales, empresas, comunidades, clases sociales e identidades grupales de muy diverso cuño, y los actores que etnografían tanto Susana Moreno y Juan Pablo Aris como Gonzalo Saavedra han de arrostrar estas dinámicas, y ser co-partícipes de las mismas.

Según Wallerstein, la estrategia de los Estados a partir de la fractura sistémica de 1968 -que significó la cristalización y profundización desde entonces de la brecha entre los territorios centrales y los periféricos-, fue la búsqueda de la globalidad y la localidad al mismo tiempo. Así quedaba desbordado, por arriba y por abajo, su marco tradicional de pensamiento y actuación (Appadurai, 1996). Ante este paisaje político se requiere la aplicación de una perspectiva sistémica, antes que estado-céntrica. Ello no implica la desaparición del Estado nacional, sino su contextualización como estructura política dentro del sistema mundial al que pertenece, y sí exige la inclusión de la perspectiva local. En este caso, de asociaciones de pescadores, de sus iniciativas, de sus formas de apropiación de discursos exógenos, o de la reutilización de sus discursos tradicionales, y tradicionalistas, en el nuevo marco. 
Los Estados se han especializado -sin excluir por ello su naturaleza política-como agentes geo-económicos, participando en reconfiguraciones territoriales para obtener una posición privilegiada de cara a los flujos económicos y comerciales emergentes (Cowen \& Smith, 2009), de modo que ya no reproducen el papel de protección a comunidades sectoriales y locales, como los pescadores artesanales en tanto que comunidades de un supuesto cuerpo nacional.

La incertidumbre y el carácter difuso y fluido de los procesos económicos y políticos afecta de pleno al tejido que seguimos concibiendo como artesanal. Desde las últimas dos o tres décadas, hay procesos que, habiendo sido característicos de los segmentos industriales, vienen afectando a las flotas artesanales, como el uso de nuevas tecnologías, la intensificación de las producciones, y su extraversión creciente a canales comerciales de carácter internacional. Ello ha obligado a repensar el concepto de pesca artesanal (Florido, 2008). Como consecuencia de todo ello, no podemos asumir de modo acrítico que las flotas artesanales mantengan, per se, dinámicas de equilibrio sistémico, en un contexto de competencia comercial creciente, tecnificación y de creciente desigualdad en las relaciones laborales. La complejidad de la articulación entre los procesos globales y locales, en cada caso, nos dibujará un escenario diferente, pero comparable, entendemos, en estas dinámicas. La artesanalización que se ha conocido en flotas como la andaluza no ha significado la vuelta a patrones de equilibrio ecosistémico y social de otros horizontes histórico-culturales.

Las etnografías presentadas en este volumen sobre Chile y Senegal nos obligan a revisar los conceptos al uso de pesca artesanal en el mismo sentido apuntado. Desde luego, como se defiende en estos trabajos, no pueden entenderse como dos fases de un proceso evolutivo. Si bien es cierto que existen indicadores que nos permiten distinguir con nitidez ambas esferas (acceso a mercados exteriores, insumos de energía y capital, grado de intensidad en el uso de la fuerza de trabajo, uso de conocimientos vernáculos, etc.); sin embargo, hay un conjunto de procesos económicos y socio-laborales que atraviesan ambos modelos, mostrando su compleja articulación. La relación con el mercado de las pesquerías ha sido histórica, si bien por canales y ámbitos territoriales diferentes, mediante distintos agentes y racionalidades culturales también diversas. También han sido históricos, en relación a las flotas artesanales, los conflictos entre modalidades, los episodios de sobreexplotación y períodos de escasez del recurso.

\section{5. ¿QUÉ ENFOQUE PARA UNA REALIDAD COMPLEJA E HÍBRIDA?}

El investigador debe dotarse de herramientas conceptuales y metodológicas sensibles a las realidades híbridas, fluidas y difusas. El trabajo de Gonzalo Saavedra ejemplifica bien la aplicación de una perspectiva de Economía Política, contextual, que da cuenta de la interrelación entre dinámicas sociales localizadas y procesos transterritoriales, de carácter global, tanto económicos como políticos. Como ya hemos reivindicado en más 
de una ocasión, se trata de adoptar un enfoque translocal, capaz de articular dinámicas globales y locales, que no pueden entenderse como esferas segmentadas, sino como la trabazón conflictiva de racionalidades culturales diversas, las cuales, en el mismo proceso de su articulación, se van transformando recíprocamente. Lógicamente, este análisis no puede llevarse a efecto sin introducir la variable poder, unas veces instrumentado desde organismos gubernamentales, en las diversas escalas de la gestión administrativa; otras veces, desde grandes corporaciones y empresas, representadas por lobbies. Lo que puede aportar el análisis etnográfico de los antropólogos es que también las sociedades locales tienen capacidad de organizar sus estrategias, desde sus valores y finalidades instrumentales, y participar, aunque con menos resultados tangibles, en el juego del poder.

Lo que el caso de las reservas marinas de interés pesquero pone de manifiesto es la inseparabilidad de las relaciones ecológicas respecto de las sociales y políticas. Como argumentan José Pascual y Raquel Modino, una figura de protección no es meramente un modo de gestionar un recurso biológico, sino fundamentalmente una forma de establecer acuerdos políticos que regulen relaciones económicas, políticas (sobre el territorio fundamentalmente) y sociales. El enfoque de la Ecología Política, por tanto, es el más adecuado para abordar este carácter multiespecífico de relaciones y procesos en juego. Parafraseando a Symes (1996), la crisis pesquera no es tanto biológica cuanto política, en el bien entendido que la solución de aquella pasa necesariamente por la toma de decisiones, por la participación dialógica de los diversos colectivos implicados, y por la puesta en comunicación de sus conocimientos y perspectivas.

Un modelo teórico acorde con esta realidad es la gobernanza reflexiva o adaptativa (Michel, 2009; Folke et al. 2005). Se trata de facilitar el encuentro de un

"broad range of actors at multiple scales to deal with the interrelated dynamics of resources and ecosystems, management systems and social systems, as well as uncertainty, unpredictability, and surprise. Adaptive governance focuses on experimentation and learning" (Folke et al. 2005: 462).

Desde este paradigma se amplía la atención a los actores y sus redes sociales para crear una estructura de decisión en la gestión mucho más densa y compleja, adaptada a los contextos particulares. Para Wallerstein (Schouten, 2008), ante la crisis del sistema mundial de finales de la primera década del s. XXI, caben dos caminos posibles: o el abundamiento en la senda de una jerarquía mundial basada en relaciones de explotación económica, dominación política y tiranía de los modelos de conocimiento occidentales; o bien, la construcción de un nuevo orden, sobre valores más igualitarios, horizontales y democráticos. Los estudios de caso aquí presentados apuestan sin duda por este segundo camino. 


\section{REFERENCIAS BIBLIOGRÁFICAS}

Agnew, John A (2003) Geopolitics: Re-visioning World Politics. London: Routledge. $2^{\text {nd }}$ Edition.

Appadurai, Arjun (1996) "Sovereignty without Territoriality: Notes for a Postnational Geography”. In P. Yeager (ed.) The Geography of Identity. Ann Arbor: University of Michigan Press, pp. 40-58.

Cowen, Deborah \& Smith, Neil (2009) "After Geopolitics? From the Geopolitical Social to Geoeconomics". Antipode, 41(1), pp. 22-48.

Florido del Corral, David (2008) "Focusing on Artisanal Fleets in a New Scenario: the Case of Andalusia (Spain)”. Marine Policy. 32 (6), pp. 1004-1012.

Folke, Carl, Hahn, Thomas, Olsson, Per \& Norberg, Jon (2005) "Adaptive Governance of Social-Ecological Systems”. Annual Review Environment and Resources, 30, pp. 441-73.

Held, David \& McGrew, Anthony (eds.) (2002) Governing Globalization: Power, Authority and Global Governance. Cambridge: Polity Press.

Jentoft, Svein; Pascual-Fernandez, Jose; De la Cruz Modino, Raquel; Gonzalez-Ramallal, Manuel y Chuenpagdee, Ratana (2012) "What Stakeholders Think About Marine Protected Areas: Case Studies from Spain”. Human Ecology, 40(2): 185-197.

Kooiman, Jan (2008) "Exploring the Concept of Governability". Journal of Comparative Policy Analysis, 10(2), pp. 171-190.

Kooiman, Jan; Bavinck, Maarten; Jentoft, Svein y Pullin, Roger (Eds.) (2005) Fish for Life: Interactive Governance for Fisheries. Amsterdam: Amsterdam University Press.

Michel, David (2009) "Foxes, hedgehogs, and greenhouse governance: Knowledge, uncertainty, and international policy-making in a warming World". Applied Energy, 86, pp. 258-264.

Power, Marcus \& Campbell, David (2010) “The state of critical geopolitics”. Political Geography, 29 (5), pp. 243-246.

Santos, Boventura de Sousa (2007) "Beyond Abyssal Thinking: From Global Lines to Ecologies of Knowledges”, Review, XXX, 1, pp. 45-89.

Schouten, P (2008) "Theory Talk \#13: Immanuel Wallerstein on World-Systems, the Imminent End of Capitalism and Unifying Social Science". Theory Talks, http://www. theory-talks.org/2008/08/theory-talk-13.html [consultado el 04-12-2013].

Symes, David (1996) "Fishing in Troubled Waters". In Crean, K \& D. Symes (Eds.) Fisheries Management in crisis. Blackwell Sciencie. Oxford: Fishing New Books, pp. 3-16.

Wallerstein, Inmanuel (2004) World-Systems Analysis: An Introduction. Duke University Press Books. 